\title{
Combining Solar Electric Propulsion and Chemical Propulsion for Crewed Missions to Mars
}

\author{
Tom Percy \\ Jacobs ESSSA Group/SAIC \\ 6723 Odyssey Dr. \\ Huntsville, AL 35806 \\ 256-544-0397 \\ Thomas.K.Percy@nasa.gov
}

\author{
Melissa McGuire \\ NASA \\ 21000 Brookpark Road M.S. 162-2 \\ Cleveland, Ohio 44135 \\ 216-977-7128 \\ Melissa.L.McGuire@nasa.gov
}

\author{
Tara Polsgrove \\ NASA \\ MSFC, FP30 \\ Huntsville, AL 35812 \\ (256) 544-1274 \\ Tara.Polsgrove@nasa.gov
}

\begin{abstract}
This paper documents the results of an investigation of human Mars mission architectures that leverage near-term technology investments and infrastructures resulting from the planned Asteroid Redirect Robotic Mission (ARRM), including high-power Solar Electric Propulsion (SEP) and a human presence in Lunar Distant Retrograde Orbit (LDRO). The architectures investigated use a combination of SEP and chemical propulsion elements. Through this combination of propulsion technologies, these architectures take advantage of the high efficiency SEP propulsion system to deliver cargo, while maintaining the faster trip times afforded by chemical propulsion for crew transport. Evolved configurations of the Asteroid Redirect Vehicle (ARV) are considered for cargo delivery. Sensitivities to SEP system design parameters, including power level and propellant quantity, are presented. For the crew delivery, liquid oxygen and methane stages were designed using engines common to future human Mars landers. Impacts of various Earth departure orbits, Mars loiter orbits, and Earth return strategies are presented. The use of the Space Launch System for delivery of the various architecture elements was also investigated and launch vehicle manifesting, launch scheduling and mission timelines are also discussed. The study results show that viable Mars architecture can be constructed using LDRO and SEP in order to take advantage of investments made in the ARRM mission.
\end{abstract}

\section{TABLE OF CONTENTS}

1 GENERAL MISSION SUMMARY.................1

2 ARCHITECTURE OVERVIEW..................... 2

3 ELEMENT OVERVIEW..............................3

4 ARCHITECTURE SENSITIVITIES................6

5 SUMMARY AND CONCLUSIONS..................9

REFERENCES......................................10

BIOGRAPHIES.....................................10

\section{GENERAL MISSION SUMMARY}

In 2014, the NASA Human spaceflight Architectures Team (HAT) conducted a study known as the Evolvable Mars Campaign (EMC) to define how near term investments could build upon one another to enable human missions to Mars. NASA's human Mars mission planning is in support

U.S. Government work not protected by U.S. copyright 2015 of the policies outlined in the 2010 NASA Authorization Act and National Space Policy and further defined in NASA's 2014 Strategic Plan. The National Space Policy directs that NASA should "By the mid-2030s, send humans to orbit Mars and return them safely to Earth." The EMC study team identified transportation architecture options supporting human missions to the Mars vicinity in the mid2030s and to the surface of Mars by the end of the 2030s.

Major ground rules of the EMC study include:

1) Leverage existing assets and current plans (ISS, Orion, SLS, the ARRM, and the Exploration Augmentation Module);

2) The campaign must be sustainable with a steady cadence of missions that will demonstrate progress in the near-term;

3) The campaign must use the "proving ground" to test and validate systems prior to a human Mars mission;

4) Designers must consider the potential use of a staging point in cis-lunar space or Earth orbit;

5) Each system developed must be evolvable to enabling a future human mission (minimize or eliminate mission unique system developments) and reusable to the greatest extent possible; and

6) Missions to Mars must employ a crew of 4 on a Mars low-energy mission.

The transportation investigation discussed in detail in this paper is one facet of the EMC study. Several potential techniques were investigated for delivering crew and cargo to Mars using elements that can be evolved from today's technology investments. In general, the EMC takes a "splitmission" approach in which mission elements are predeployed to the destination using propulsion techniques that are more mass-efficient, but may require transit times longer than those desirable for crewed flight. Because the delivery of these elements (Mars surface landers and even the propellant stages needed for crew return) is less time dependent, they can be pre-positioned in Mars orbit utilizing low-thrust trajectories and Solar Electric Propulsion (SEP), while also spreading launches over a longer period of time. The crew and their transit elements can then be sent on a high-thrust trajectory from a cis- 
Lunar staging point to Mars orbit, minimizing crew mission time. This implies a different risk posture than traditional mission techniques, as remote rendezvous and integration of these pre-deployed elements is required for mission success and perhaps even crew survival, but it remains true to the goals of the EMC by leveraging today's investments to complete tomorrow's most challenging mission.

\section{ARCHITECTURE OVERVIEW}

The architecture discussed in this paper stays consistent with the intent of the EMC study by combining near-term investments in the development of Solar Electric Propulsion (SEP) while leveraging planned future developments in chemical propulsion to send humans to Mars in the 2030s. This SEP-Chem architecture draws on the strengths of each technology to perform different yet complimentary functions required for humans to explore Mars. The low-thrust SEP spacecraft is used to pre-position elements such as habitats, orbital maneuvering systems, consumables, and landers for use in the Martian system. The Chemical propulsion stages deliver the crew using high-thrust trajectories that minimize trip time to the extent possible to support crew health and safety. Together, these elements comprise the building blocks of a sustainable human Mars exploration campaign.

The Mars campaign initially consists of 3 sequential missions. The first is an orbital mission during which the crew will explore the Martian moon of Phobos. The second mission will deliver people to the surface of Mars for the first time. This first landing mission will support only a short stay on the Martian surface but will begin the buildup of resources required for subsequent missions with longer surface durations. While only spending a short time on the Martian surface, the crew will still remain in the Mars system for the duration of a long-stay mission ( $\sim 500$ days) and will perform other scientific investigations while in orbit. In the third mission of the sequence, a full crew will return to the landing site of the previous mission with a full complement of resources and equipment necessary to complete a long surface stay, becoming the first humans to spend more than 1 year on the surface of a planet other than Earth.

A campaign of this magnitude requires the delivery of many different elements to Mars. Equipment for exploring Phobos includes a habitat and crew transfer vehicles necessary for maneuvering within the Mars system. Landers carrying a suite of surface assets, including habitats, surface power systems, rovers, and the 2-stage Mars Ascent Vehicle (MAV), must also be delivered to Mars to support human landing missions. Finally, the crew itself, housed in an exploration habitat and carrying with it enough consumables and support equipment to sustain themselves over the nearly 1000 day mission, must also be delivered to Mars and safely returned to Earth. It is the goal of the transportation team to design propulsion elements and trajectories to support this wide array of delivery requirements.

Each mission to Mars can be divided into a cargo predeploy phase and a crew delivery phase. During the cargo pre-deploy phase, an Space Launch System (SLS) 2B launch vehicle is used to deliver integrated SEP-payload stacks to elliptical Earth orbits. From there, each integrated payload stack uses the SEP system to spiral out of the Earth's gravity well, travel through interplanetary space, and spiral down into the desired Mars parking orbit. All of the human flights to Mars in this architecture park in a 1 Sol Mars orbit, taking advantage of the higher orbital energy to reduce the energy requirements on the chemical propulsion system that delivers the crew. Any predeployed assets that the crew will use must be delivered to this same 1 Sol orbit. For the Phobos exploration mission, some pre-deployed elements must also be delivered to Phobos, requiring flexibility in the design of the SEP system to be capable of traveling to multiple Mars destinations. Figure 1 shows a simplified version of this pre-deploy sequence.

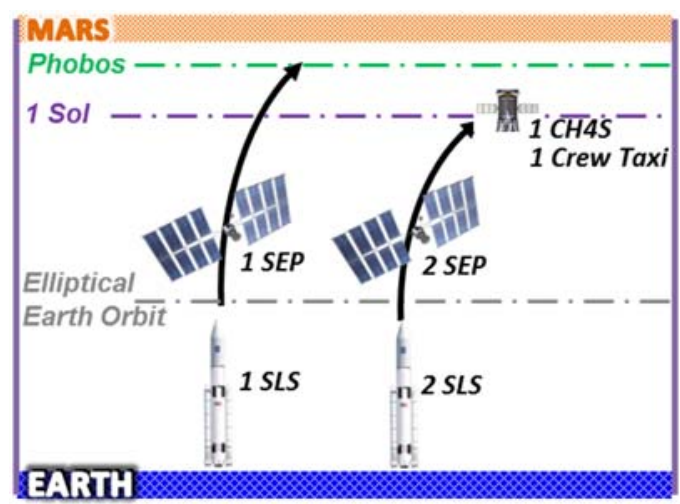

Figure 1: A simplified view of the Cargo Pre-Deploy Phase of the SEP-Chem Architecture

The crew delivery phase uses high-thrust, chemical propulsion to deliver the crew on a more traditional trajectory to Mars. Again, the SLS 2B launch vehicle is employed to deliver mission elements to orbit, however in this mission phase, the propulsive elements and crew transfer habitat are delivered to the Lunar Distant Retrograde Orbit (LDRO). This aggregation orbit builds on infrastructure already in place as a result of the ARM mission and provides a stable outpost for collecting and assembling the elements of the crew stack prior to Earth departure. Once assembled, the stack provides a small impulse to perform a low delta-v transfer to a Lunar Distant High Elliptical Orbit (LD-HEO) which has a LEO perigee and a near-lunar distance apogee. The crew is launched on a crew version of the SLS into this LD-HEO where they rendezvous with the crew stack and prepare for the transit to Mars. Using this high orbit reduces the energy requirement for Trans-Mars Injection (TMI), an advantage 
that will be further discussed in the Crew Trajectory Analysis section of this paper.

Another unique aspect of this architecture is that, unlike previous Mars architectures, the crew stack Earth return propulsion is pre-deployed using SEP and is waiting for the crew in $1 \mathrm{Sol}$ orbit. This further reduces the size of the crew stack and takes advantage of the high-efficiency of the SEP to deliver non-time critical elements of the mission. Therefore, once the crew stack has performed the Mars Orbit Insertion (MOI) burn, the MOI stage is dropped and the Earth return elements are mated to the transfer habitat. After performing their mission at Mars for the prescribed long-stay duration (350-550 days), the crew returns to Earth using these pre-deployed assets.

This SEP-Chem architecture further capitalizes on the unique aspects of the LD-HEO Earth parking orbit and the pre-deployment of Earth return assets to perform a propulsive capture upon returning to Earth. In most traditional Mars missions, the crew stack is so large that placing the additional burden of Earth orbit capture at the end of the mission on the stack is big a challenge. Therefore, these missions assume a direct re-entry and carry the re-entry vehicle all the way to Mars and back. Given the uniquely low energy requirement of capture into the LD-HEO, the SEP-Chem architecture performs a propulsive capture at Earth, meets with a freshly delivered Orion capsule, and the crew re-enters under significantly less strenuous conditions than on a direct entry.

This maneuver enables three major advantages over traditional missions. First, it avoids the requirement of carrying a re-entry vehicle through the 1000 day round-trip journey to Mars. By using a fresh Orion capsule, reliability is increased. Second, it avoids the requirement to upgrade the thermal protection system on the Orion capsule. The baseline Orion TPS is designed for re-entry from the Moon, essentially identical conditions to those experienced on reentry from the LD-HEO. Traditional Mars missions require upgrades to the Orion TPS to support re-entry at significantly higher speeds. The extended quiescent period and higher entry velocities lead to a requirement for a "Mars Orion". The SEP-Chem architecture can be executed with the standard Orion. Finally, the propulsive capture at Earth return enables the reuse of the transfer habitat. Once the crew has left the stack in LD-HEO, another low energy transfer sends the transfer habitat back to LDRO where it can be repaired, restocked, and readied for the next flight to Mars. Propulsive capture at Earth does, however, require a final, critical burn which will impact loss-of-crew probability assessments and puts some additional burden on the Earth return stage for prolonged life and long periods of time between main engine ignitions. Figure 2 shows a simplified version of the crew flight sequence.

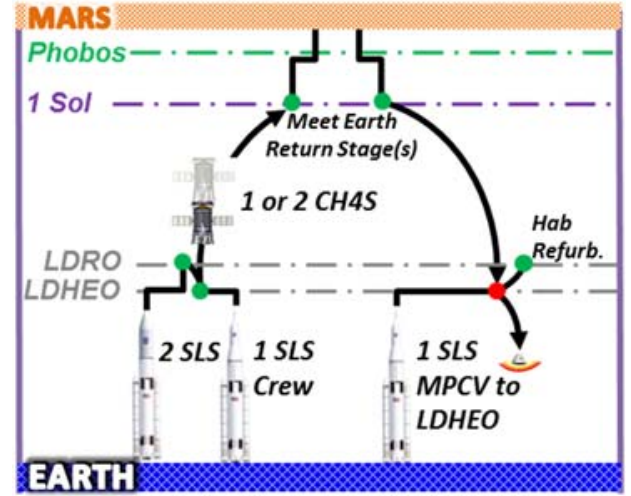

Figure 2: A simplified view of the Crew Delivery Phase of the SEP-Chem Architecture

\section{ELEMENT OVERVIEW}

\section{SEP Element Description}

After performing a series of power and propellant trades, the cargo delivery performance analysis focused on an SEP stage that was deemed extensible to the Asteroid Robotic Redirection Mission (ARRM). This evolved SEP stage was examined in detail. This stage assumed $150 \mathrm{~kW}$ of power to the EP thruster system, and a limit of $16 \mathrm{t}$ of xenon (Xe) propellant. Based on the ARRM $40 \mathrm{~kW}$ vehicle, this $150 \mathrm{~kW}$ stage shared the same bus and thruster system design. The variations from the ARRM design are in the solar array area, mission module power requirements to the payload, and number of thrusters. A picture of the $150 \mathrm{~kW}$ SEP stage is provided in Figure 3.

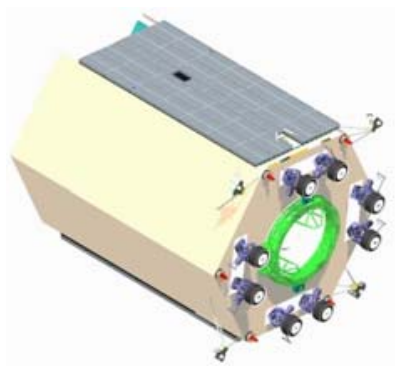

Figure 3: The SEP Stage

The $150 \mathrm{kWe}$ SEP Stage is extensible from the current ARRM SEP stage design, using the same spacecraft bus as the current $40 \mathrm{~kW}$ to the EP system concept ARV. The dry mass assumptions for the SEP stage were scaled using a relationship derived from preliminary ARV designs, which varied total dry mass with total power to the EP thruster system and total Xe propellant mass. With an augmented propellant capability of $16 \mathrm{t}$, this SEP stage is launched with its integrated payload on a single SLS launch to an elliptical starting orbit. The SEP Stage will spiral the payload to a $\mathrm{C} 3$ of $-2 \mathrm{~km}^{2} / \mathrm{s}^{2}$ in order to line up for a Lunar Gravity Assist (LGA). Post LGA, the SEP stage starts an interplanetary trajectory with a $\mathrm{C} 3$ of $2 \mathrm{~km}^{2} / \mathrm{s}^{2}$ and spirals in heliocentric space to Mars vicinity. Using a single launch, the $150 \mathrm{~kW}$ stage can deliver $30-40 \mathrm{t}$ payloads to the 
various Mars destinations currently under examination. Table 1 below captures the specific design parameters of the $150 \mathrm{~kW}$ SEP stage examined in detail in this analysis.

Table 1: SEP Design Parameters/Constraints

\begin{tabular}{|l|l|}
\hline \multicolumn{2}{|c|}{150 kW SEP Stage Design Parameters/Constraints } \\
\hline Engine Technology & Magnetically Shielded Hall \\
\hline Engine Isp & $3000 \mathrm{Sec}$ \\
\hline Power to EP system & $150 \mathrm{~kW}\left(1 / \mathrm{R}^{2}\right.$ for R>1AU) \\
\hline Thruster Efficiency & $60 \%$ \\
\hline Thruster Duty cycle & $90 \%$ \\
\hline Propellant & Xe (limited to 16 t) \\
\hline Solar Array Technology & MegaFlex (shown) \\
\hline Number of wings & 2 \\
\hline Wing diameter & $18.7 \mathrm{~m}$ \\
\hline Power per wing & $75 \mathrm{~kW}$ \\
\hline
\end{tabular}

\section{SEP Trajectory Analysis}

The high efficiency of an SEP system lends the technology to the application of delivering cargo to interplanetary destinations such as Mars. Solar Electric Propulsion makes use of the conversion of solar power to electrically accelerate propellant to higher energies. SEP enables delivery of significant payloads even at near term power levels, given enough trip time. For the Mars mission architecture, a single SLS launch of the SEP Stage and its cargo to Mars was assumed. The SLS launched the SEP stack to a Low Earth Orbit (LEO) where the SEP stage performed a spiral trajectory targeting a lunar gravity assist (LGA). After LGA to Earth escape, the SEP stage performed an interplanetary spiral trajectory to mars, where it completed its mission by spiraling to a final Mars capture orbit. For this analysis, four orbits were examined as options for Mars cargo destinations: Low Mars orbit (500 km circular), 1 Sol, at Phobos orbit, and at Deimos orbit. Figure 4 below depicts the minimum time Earth to Mars interplanetary trajectory using the $1 / \mathrm{R}^{2}$ power law for the solar array power to the thrusters.

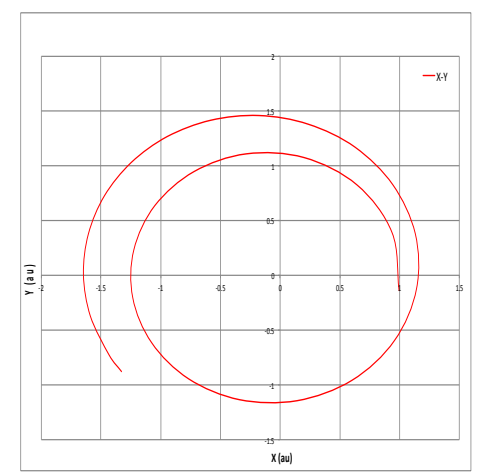

Figure 4: SEP heliocentric spiral from Earth to Mars

\section{SEP Cargo Delivery Performance}

Table 2 below captures the minimum flight time payload delivery performance capability of the $150 \mathrm{~kW}$ SEP stage. In all cases, the trajectory analysis used the entire assumed limit of $16 \mathrm{t}$ of Xe propellant. The 1 Sol and Phobos delivered payload is a minimum time solution. Given slightly more time in interplanetary space, additional payload capabilities can be captured. For the aerocapture solution, the trajectory analysis used a goal of $41 \mathrm{t}$ delivered to an arrival $\mathrm{V}_{\infty}$ of $7.4 \mathrm{~km} / \mathrm{s}$. For the aerocapture case, the SEP stage only required $11.7 \mathrm{t}$ of $\mathrm{Xe}$ to perform the mission. Therefore, the SEP stage is capable of delivering more given more time either in interplanetary space or during the Earth escape spiral.

Table 2: SEP Stage Minimum Flight Time Performance Summary

\begin{tabular}{|l|c|c|c|c|}
\hline Destination & $\begin{array}{c}\text { SEP dry } \\
\text { mass }(\mathrm{t})\end{array}$ & Xe (t) & $\begin{array}{c}\text { Delivered } \\
\text { payload (t) }\end{array}$ & $\begin{array}{c}\text { Total trip } \\
\text { time }(\mathrm{yr})\end{array}$ \\
\hline 1 Sol & 7.68 & 16 & 31 & 4.6 \\
\hline Phobos & 7.68 & 16 & 29 & 4.8 \\
\hline Aerocapture & 7.75 & 11.7 & 41 (target) & 2.8 \\
\hline
\end{tabular}

\section{Methane Stage Description}

While the high efficiency of an SEP system is excellent for delivering cargo to Mars, long trip times associated with low-thrust trajectories are undesirable for crew delivery. The SEP-Chem architecture uses a high-thrust chemical propulsion system to deliver the crew on a more traditional trajectory to Mars. The in-space propulsion staged used in this architecture is a liquid oxygen / liquid methane $\left(\mathrm{LOx} / \mathrm{LCH}_{4}\right)$ stage based on the engines that will be developed for the crewed Mars landers. Figure 5 is a drawing of the methane stage.

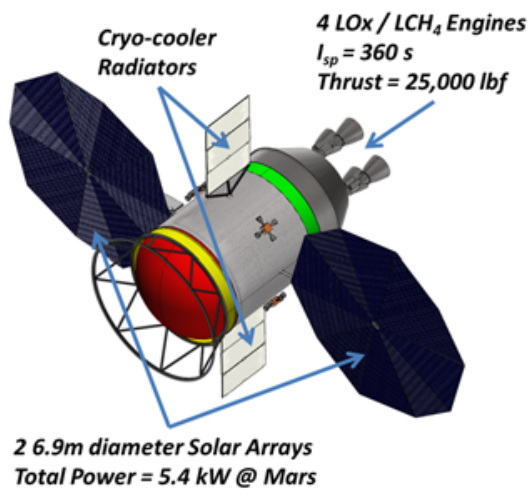

Figure 5: The Methane Stage

In many previous Mars transportation studies, the propellant of choice has been a combination of liquid oxygen and liquid hydrogen $\left(\mathrm{LOx} / \mathrm{LH}_{2}\right)$. The justification has been that a higher specific impulse is required to manage the size of the stages required for such a high energy mission as a transit to Mars. There are, however, significant technology challenges related to the longduration storage of liquid hydrogen. Two unique aspects of the SEP-Chem mission, the pre-deploying of Earth return propulsion to reduce the mass of the crew stack at Earth departure and the use of the LD-HEO, reduce the 
overall energy requirement of the TMI burn to a level achievable by a system with a specific impulse lower than 465 seconds (the typical value for a $\mathrm{LOX} / \mathrm{LH}_{2}$ stage). This enables the use of a 360 second specific impulse $\mathrm{LOx} / \mathrm{LCH}_{4}$ system and avoids developing the cryo-cooler technology required to support long duration liquid hydrogen storage.

The ability to use the $\mathrm{LOx} / \mathrm{LCH}_{4}$ engines provides several other benefits. The stage designed for this architecture uses the $\mathrm{LOx} / \mathrm{LCH}_{4}$ engines that will be used on the crewed Mars landers. This provides a common development path for both propulsive elements. Long-duration storage of cryogenic propellants such as $\mathrm{LOx}$ and $\mathrm{LCH}_{4}$ still requires technology development; however this technology is less challenging than the technology required for $\mathrm{LH}_{2}$ storage. A common cryogenic fluid management (CFM) system will also be developed for both the lander and the in-space stage.

The methane stage has a diameter of 4 meters and a length of 10 meters. The tanks are constructed of 2195 aluminum in a simple-to-manufacture monocoque configuration while the dry structure is 2219 aluminum. The power system uses two $6.9 \mathrm{~m}$ diameter UltraFlex solar arrays to provide $5.4 \mathrm{~kW}$ of electrical power at Mars. The majority of this power is required for cryogenic propellant storage. Deployable radiators are also designed to support the heat rejection from the CFM system. The CFM system employs $90 \mathrm{~K}$ cryo-coolers for the storage and conditioning of both the $\mathrm{LOx}$ and the $\mathrm{LCH}_{4}$.

The stage is designed with a full complement of guidance, navigation, and control sensors to support free-flying time during the assembly phase of the mission. This provides a level of redundancy for stacks with multiple stages and ensures that minimal stage customization will be required during manufacturing. The main propulsion system is made up of four $111,000 \mathrm{~N}$ (25,000 lbf) methane engines, common to the Mars lander. The Reaction Control System uses pressurized $\mathrm{LOx} / \mathrm{LCH}_{4}$, providing a common propellant and avoiding the use of traditional hypergolic propellants that require additional safety procedures for loading. A mass statement for the methane stage is provided in Table 3 below.

Table 3: The Methane Stage Mass Statement

\begin{tabular}{|c|c|c|c|c|}
\hline MEL - Mars CH4 & Basic Mass (kg) & Contingency $(\%)$ & Contingency (kg) & Predicted Mass (kg) \\
\hline \multicolumn{5}{|l|}{ Mass Breakdown Structure } \\
\hline $1.0 \mid$ Structures & 2004.36 & $25.00 \%$ & 501.09 & 2505.45 \\
\hline \begin{tabular}{l|l|l|l|l}
2.0 & Propulsion \\
\end{tabular} & 1963.57 & $25.00 \%$ & 490.89 & 2454.46 \\
\hline 3.0 Power & 746.00 & $15.00 \%$ & 111.90 & 857.90 \\
\hline 4.0 Avionics & 748.25 & $17.62 \%$ & 131.85 & 880.10 \\
\hline \begin{tabular}{l|l|}
5.0 & Thermal \\
\end{tabular} & 646.87 & $16.46 \%$ & 106.50 & 753.37 \\
\hline Dry Mass & 6109.05 & $21.97 \%$ & 1342.23 & 7451.28 \\
\hline \begin{tabular}{|c|c|}
6.0 & Non-Prop Fluids \\
\end{tabular} & 1511.19 & & & 1511.19 \\
\hline Inert Mass & 1511.19 & & & 1511.19 \\
\hline Total Less Propellant & 7620.24 & & & 8962.47 \\
\hline \begin{tabular}{|l|l|}
$7.0 \mid$ Usable Propellant \\
\end{tabular} & 30873.40 & & & 30873.40 \\
\hline Total Stage Gross Mass & 38493.64 & & & 39835.87 \\
\hline
\end{tabular}

\section{Methane Stage Trajectory Analysis}

Interplanetary trajectory analysis for the high-thrust crew delivery phases of the mission was completed using the JPL-developed Mission and Design and Analysis Software (MIDAS). For each flight opportunity from 2033 to 2041, the minimum energy trajectory for a roundtrip was found through optimization using MIDAS. Figure 6 shows the crew trajectory for the 2033 flight as an example. Note that this trajectory includes a deep space maneuver to account for misalignment between the interplanetary trajectory and the Earth departure hyperbola.

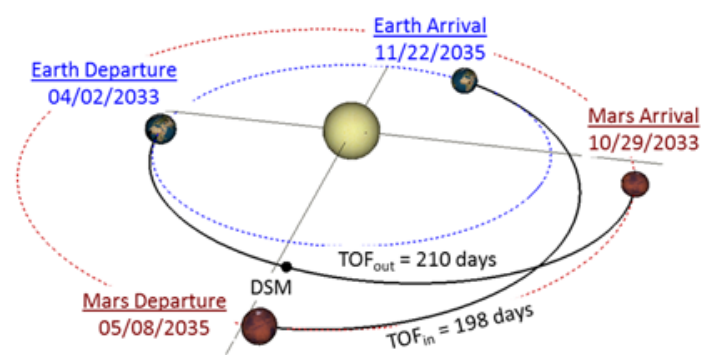

Figure 6: High-Thrust Conjunction-Class Transfer Trajectory for Crew Delivery to Mars

Table 4 provides the main propulsion $\Delta \mathrm{V}$ budget for each flight opportunity. The Trans-Mars Injection (TMI) and Earth Orbit Insertion (EOI) burns are assumed to occur at perigee of our Lunar Distant High Elliptical Orbit. This results in significantly lower $\Delta \mathrm{V}$ requirements for these maneuvers when compared to a traditional Mars mission which departs Earth from a $407 \mathrm{~km}$ circular orbit.

Table 4: High-Thrust Trajectory $\Delta \mathrm{V}$ Budget
\begin{tabular}{|c|c|c|c|c|}
\hline Earth & \multicolumn{4}{|c|}{$\Delta \mathrm{V}$ (including $4 \%$ FPR) $(\mathrm{km} / \mathrm{s})$} \\
Departure Date & TMI & MOI & TEI & EOI \\
\hline $4 / 2 / 2033$ & 1.069 & 1.180 & 1.053 & 0.528 \\
\hline $6 / 25 / 2035$ & 0.575 & 0.902 & 1.496 & 0.548 \\
\hline $8 / 18 / 2037$ & 0.849 & 0.966 & 1.126 & 0.482 \\
\hline $9 / 23 / 2039$ & 0.665 & 0.854 & 0.951 & 0.493 \\
\hline $10 / 5 / 2041$ & 0.660 & 1.033 & 1.101 & 0.537 \\
\hline
\end{tabular}

Table 5 outlines the trip times for each leg of each flight to Mars. Trip time was used in this analysis to size the consumables loaded into the crew transit habitat. While the total trip times for all of the missions in the set were in the typical 1000-day range, it is important to note that the minimum energy trajectories in the later years in the cycle require significantly more interplanetary time and result in significantly shorter Mars stay times. 
Table 5: High-Thrust Trajectory Trip Times

\begin{tabular}{|c|c|c|c|c|}
\hline Earth & \multicolumn{4}{|c|}{ Trip Time (days) } \\
Departure Date & Outbound & Stay & Return & Total \\
\hline $4 / 2 / 2033$ & 210 & 556 & 198 & 964 \\
\hline $6 / 25 / 2035$ & 198 & 538 & 268 & 1005 \\
\hline $8 / 18 / 2037$ & 351 & 353 & 286 & 990 \\
\hline $9 / 23 / 2039$ & 350 & 329 & 298 & 977 \\
\hline $10 / 5 / 2041$ & 295 & 369 & 339 & 1003 \\
\hline
\end{tabular}

While the crew stacks depart Earth from the LDHEO orbit, assembly of the habitat and propulsive elements into the crew stack occurs at the LDRO orbit. This highly stable lunar orbit was selected for aggregation based on commonality with the ARRM mission, which plans to return an asteroid to the LDRO for inspection by crewed missions in 2025. Maneuvering directly into and out of the LDRO takes only a few days and requires a relatively low $\Delta \mathrm{V}$ budget if the trajectory includes a powered lunar swing-by maneuver. However, this $\Delta \mathrm{V}$ budget can be reduced to a very small number through the use of a novel multi-lunar gravity assist maneuver which moves the stack from the LDRO to the LD-HEO. An example of this maneuver is provided in Figure 7.

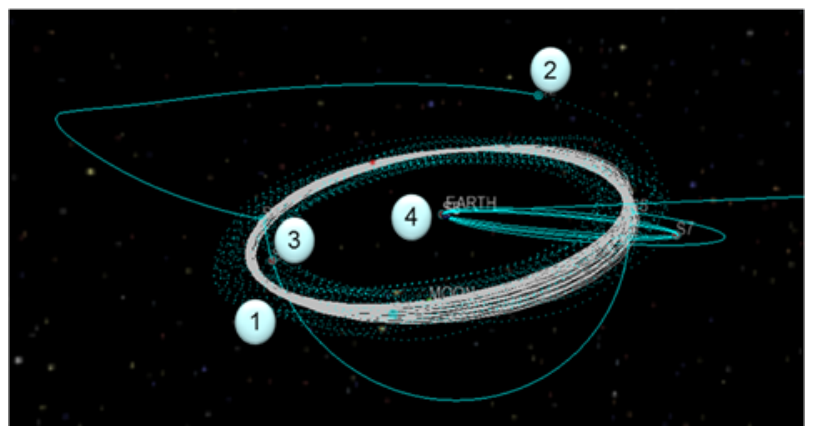

Figure 7: Low Energy LDRO-to-LDHEO Transfer Maneuver using multiple Lunar Gravity Assists

The maneuver consists of 4 small burns rather than the typical 2 burn departure directly from LDRO. The first burn is performed for the stack to depart LDRO and sets up the first Lunar Gravity Assist (LGA). Burns 2 and 3 are very small LGA targeting burns. The final burn in the maneuver is a perigee burn to target the desired LD-HEO. Instead of expending $322 \mathrm{~m} / \mathrm{s}$ as in the direct LDRO departure maneuver, the 4-burn maneuver expends only $159 \mathrm{~m} / \mathrm{s}$ of $\Delta \mathrm{V}$ and relieves the requirement to perform an LDRO insertion maneuver from the Orion flight that will meet the stack in LD-HEO to deliver the crew to their waiting Mars transfer stack. This 4-burn maneuver does take $\sim 230$ days to complete, which adds time to the overall assembly timeline, but this additional time is without the crew on board so it poses no additional risk to crew health and safety. This 4-burn maneuver was used on all mission trades evaluated in the SEP-Chem architecture analysis.

\section{Methane Stage Delivery Performance}

The performance of the methane stages in delivering crew to Mars is measured in terms of how many stages are required to deliver the habitat and supporting equipment for a given flight opportunity. The intent of the methane stage design was to minimize customization during manufacturing and, therefore, a common stage was used for all maneuvers in all crew stacks in the campaign. When a maneuver requires less propellant than the methane stage can hold, the tanks of that stage are not fully loaded. Therefore, a known payload mass must be delivered to Mars in a given flight opportunity using one or more of the Methane stages described above.

Over the flight opportunities investigated, only the 2035 opportunity requires separate stages for Trans-Earth Injection (TEI) and Earth Orbit Insertion (EOI). This particular opportunity would, therefore, require a second SEP cargo delivery flight to deliver the second methane stage for crew return. In opportunities 2035 and 2039 the Trans-Mars Injection (TMI) and Mars Orbit Insertion (MOI) maneuvers can be completed by a single methane stage. All other opportunities require two methane stages, one for TMI and a second for MOI. Table 6 provides a count summary for the number of methane stages required for a given flight opportunity. Note that the stages listed under "Return" are pre-deployed using the SEP cargo flights outlined previously.

Table 6: Number of Methane Stages Required for
Crew Delivery by Flight Opportunity
\begin{tabular}{|c|c|c|}
\hline Earth & Methane Stage Count \\
Departure Date & Outbound & Return \\
\hline $4 / 2 / 2033$ & 2 & 1 \\
\hline $6 / 25 / 2035$ & 1 & 2 \\
\hline $8 / 18 / 2037$ & 2 & 1 \\
\hline $9 / 23 / 2039$ & 1 & 1 \\
\hline $10 / 5 / 2041$ & 2 & 1 \\
\hline
\end{tabular}

\section{ARCHITECTURE SENSITIVITIES}

\section{SEP Flight Sensitivities}

Analysis of varying input power to the thruster system and propellant limits was performed on the application of a Solar Electric Propulsion system using Hall thrusters (electrostatic propulsion systems) to deliver payload to a selection of Mars orbit destinations.

In order to focus on SEP capabilities that are extensible to the technology being developed for the ARRM, the SEP Cargo analysis examined performance to mars as a function of thruster power levels starting from the $40 \mathrm{~kW}$ ARRM power upward to $300 \mathrm{~kW}$. In addition, Earth escape and interplanetary trip times were varied to find an optimal combination of delivered mass and initial SLS payload 
mass for the varying power levels. In order to limit the initial trade space, the Earth spiral time from SLS initial orbit to Earth escape was limited to one year. Additional spiral time will result in more mass delivered to Earth escape. In addition, a Lunar Gravity Assist (LGA) was added to the escape trajectory in order to increase the Earth departure energy at no propellant cost.

For all power levels, increased launch vehicle performance allows for more starting mass, and results in more payload delivered. Both the SLS $1 \mathrm{~B}$ and $2 \mathrm{~B}$ were examined as launch vehicle options, but the $2 \mathrm{~B}$ with its larger performance capability was ultimately the focus. Figure 8 below shows the SLS 2B starting orbits as a function of the SEP stage power to the EP system.

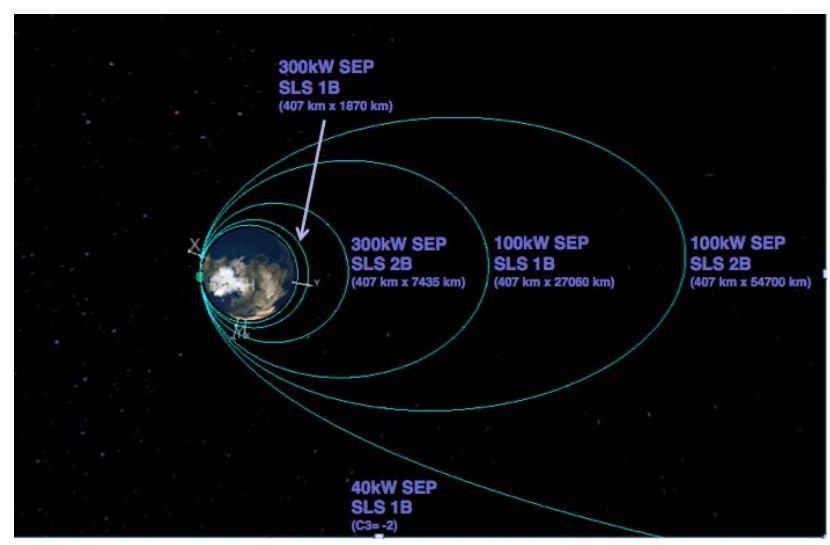

Figure 8: Starting LEO as a function of SEP power

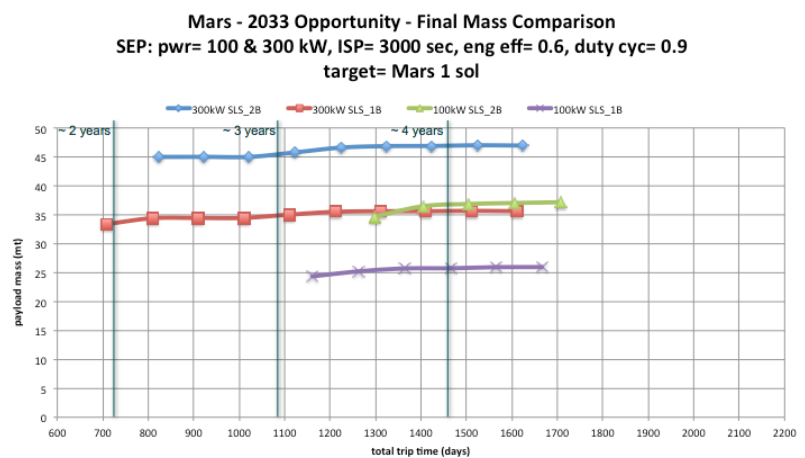

Figure 9: Delivered payload as a function of total trip time for varying power levels

Performance as a function of total trip time was examined for varying power levels and SLS 1B and 2B as shown in the graphic in Figure 9. In all cases, the more mass that can be delivered to Earth escape, the more mass that will be delivered to Mars. Anything that can be done to maximize the Earth departing mass should be examined. For these analyses, the items that increase departing mass are: larger launch vehicle performance, inclusion of a LGA, and higher power EP systems. Figure 9 below shows an example of one of the trades of payload delivered to Mars
1 Sol orbit vs total trip time. The SLS 1B using a $300 \mathrm{~kW}$ SEP stage delivered the same performance to Mars as the SLS 2B with a $100 \mathrm{~kW}$ SEP stage, although in less total trip time.

After analysis of the impact of SLS performance, Earth spiral escape, interplanetary trip time, EP system power levels and limitations on the Xe propellant capacity of the ARV extensible vehicle on delivered payload mass, the focus turned to the performance of a $150 \mathrm{~kW}$ SEP Stage.

\section{Chemical Architecture Sensitivities}

Several architecture sensitivities and trades were performed in support of the development of the SEP-Chem architecture. Two major trade evaluations were the impact of propulsive Earth return versus the traditional direct entry, and the impact of using common methane stages rather than resizing each stage for its individual contribution to the mission. A sensitivity analysis was also performed related to the payload mass delivered on allchemical crew flights. While initial mass required in Earth orbit (IMLEO) has traditionally been the metric of choice for evaluating the performance of various Mars mission architectures, our team has decided instead to focus on the number of SLS launches required. While this appears to be only a small difference, translating IMLEO into number of launches better characterizes the actual cost and risk associated with delivering that mass from the surface of the Earth to space. The number of launches metric also accounts for packaging challenges and their impact to program success. The general premise is that if an architecture option reduces the mass requirement but does not also reduce the number of launches there will ultimately be no impact to the program.

One of the most significant ways in which the use of LDHEO and LDRO changes the SEP-Chem architecture is by reducing the energy requirement upon Earth return to a level that propulsive capture is enabled. This allows for the reuse of the crew transportation habitat and avoids the modification of the Orion spacecraft for use in the Mars architecture. However, previous studies have shown significant savings for direct crew re-entry upon return to Earth. Therefore, our team investigated the impacts of propulsive capture at Earth. Both the 2035 and 2037 opportunities were investigated as they represent two unique architectures. In 2035, the TMI and MOI burns are completed with a single methane stage while 2 separate stages are required to perform the TEI and EOI maneuvers. In 2037, while the TEI and EOI maneuvers can be completed with one stage, separate stages are required for the TMI and MOI burns.

In both cases, the direct entry version of the mission was evaluated. While this version of the mission eliminates the EOI burn, which simplifies the pre-deployment phase of the mission, it does require that the $15.8 \mathrm{t}$ Orion system be 
flown to Mars and back. In the 2035 opportunity, eliminating the EOI maneuver reduces the number of methane stages pre-deployed to 1; just the TEI stage. However, the added work load of delivering the Orion spacecraft to Mars increases the size of the TMI/MOI stage beyond the lift capacity of the SLS to LDRO, thus requiring that the stage be split into two stages, one to perform TMI and another to perform MOI. The overall comparison shows that both options require the same number of SLS launches. In 2037, a similar result is observed. While the additional mass requirement of Orion requires the TMI and MOI stages to grow, they remain within the delivery limits of SLS. For Earth return, the 2037 architecture already uses only one stage to perform the TEI and EOI maneuvers. Therefore, even though the EOI maneuver is eliminated in the direct entry option, the stage count and SLS launch count remain unchanged. A comparison of the wet mass of the three stages for each of these two scenarios is provided in Figure 10.

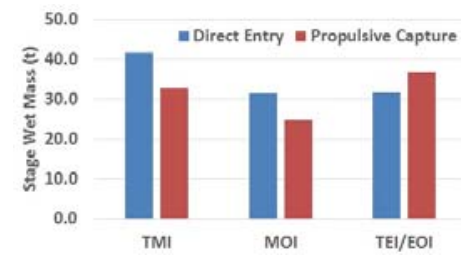

Figure 10: Stage Wet Mass Comparison for Direct Entry vs Propulsive Return in 2037

This trade shows that the number of SLS launches is not impacted by trading direct entry against propulsive capture. While previous studies have shown a distinct advantage to direct entry, these studies have typically employed a Low Earth Orbit for aggregation. By increasing the apogee of the parking orbit to lunar distance, the energy requirement for orbit capture is significantly reduced. The energy balance, in this case, is restored through the use of a large launch vehicle that can deliver the re-entry vehicle to the high Earth orbit. With performance impacts minimized, other factors can be considered. Propulsive capture offers programmatic benefits such as habitat reuse and the use of the standard Orion spacecraft that make it an appealing option.

Another architecture decision that had programmatic roots was the use of the common methane stage for all highthrust maneuvers. The general idea behind this approach is that by employing stages that are carbon copies, the manufacturing process becomes simpler and costs are reduced. The resulting condition imposed on the stages is that many of them will have some reduced propellant load and, therefore, increased propellant tank ullage. This also means that many of the stages are slightly oversized for their job resulting in some amount of unnecessary inert mass being carried throughout the mission. While this is sub-optimal from a performance standpoint, there are potential programmatic benefits to be considered. A trade was performed to determine the performance impact resulting from this programmatic assumption.

Each methane stage is sized based on a scaling equation developed during the detailed design work that generated the methane stage design discussed previously. In the common stage approach, the stage with the largest propellant load is used to set the stage dry mass for all other stages in the architecture. In this sensitivity analysis, an alternative approach allowed each methane stage to be sized independent of the others based on its individual propellant load. The analysis showed that no additional launches were required to accommodate the common stage approach. On average, each flight opportunity absorbs a 17.9t total wet mass penalty for using the common stage approach.

Taking the stage commonality assumption to the next level, a truly globally common stage is one designed to operate across flight opportunities in a given cycle. Figure 11 shows the wet mass of the stages required for each crew flight opportunity investigated. These stages are sized to be common within a particular flight opportunity but not across all opportunities. The broad orange bar at the base of each data set is the common stage dry mass.

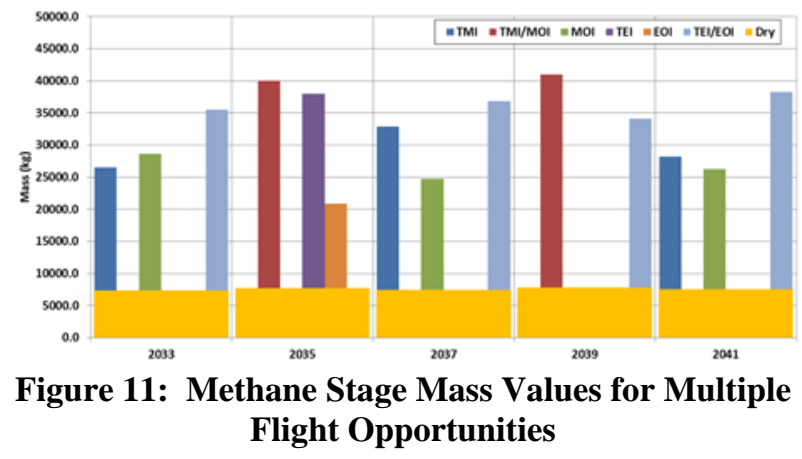

This data shows that there is very little variability in the dry mass of the common stages from opportunity to opportunity. The largest stages in each flight are nearly the same size. Therefore, a globally common methane stage can be sized using the 2039 flight opportunity and can be used across all flight opportunities with minimal impact to the overall performance.

Using a common stage approach does result in some propellant offloading in many of the stages used in the architecture. Figure 12 shows a histogram of the percent propellant offload across all of the flight opportunities investigated. The data shows that $35 \%$ of the stages require greater than $30 \%$ propellant offload. This indicates a potential for a two-common-stage approach that may reduce overall offload impacts with minimal impact to manufacturing. 


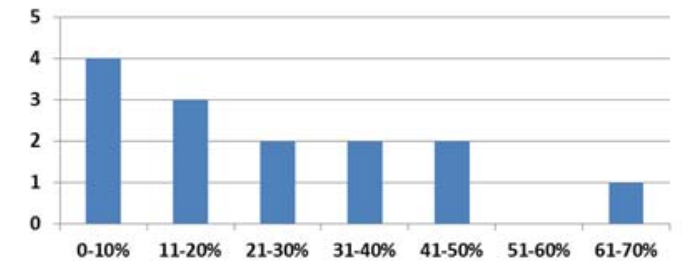

Figure 12: Histogram of Propellant Offload Percentage for Globally Common Methane Stages Across Five Flight Opportunities

Another significant driver in the sizing of the chemical propulsion element for crew delivery is the size of the transit habitat. A sensitivity analysis was completed by varying the crew stack payload mass parametrically in order to determine the impact of variations in this habitat mass as the design is further refined. In this paper, we will discuss the sensitivity in the first flight opportunity of 2033. The transit habitat element was assumed to have a mass of 36.6t based on on-going design work and the 964 day duration of this particular flight opportunity. As previously discussed, this flight opportunity requires the pre-deployment of one chemical propulsion stage to perform the Earth return and Earth orbit insertion burns as well as two chemical stages for the Earth-to-Mars leg of the mission. Thus, the total number of SLS launches required to fly the transit habitat roundtrip is 4 , resulting in a total initial mass in space of $127 \mathrm{t}$.

In this sensitivity analysis, the mass of the transit habitat was varied parametrically and the resulting mass of the chemical propulsion stages was tracked. As the mass of the habitat is reduced, stages begin to get smaller. At some point, the habitat mass is small enough to enable the transition to a single stage to perform both the TMI and MOI burns in the architecture, thus resulting in a reduction in the number of SLS launches by one. Similarly, as the habitat mass is increased, the stages begin to get larger. At some larger habitat mass, the stages grow too larger for the SEP system to deliver to Mars and the functions of TEI and EOI must be split into two stages, thus resulting in an increase in the number of SLS launches by one. This continuum of mass data and the resulting number of SLS launches are plotted for the 2033 flight opportunity in Figure 13.

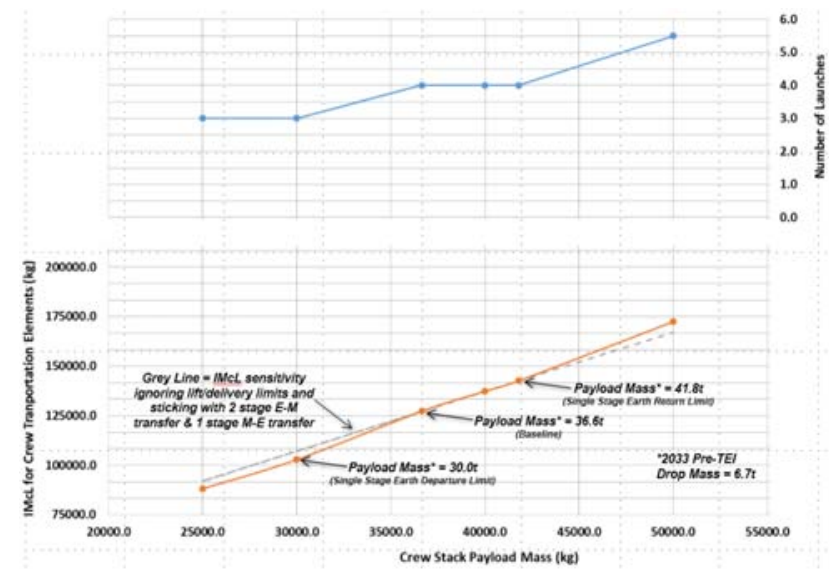

Figure 13: Architecture Sensitivity to Crew Stack Payload Mass for the 2033 Flight Opportunity

In this exercise, the habitat mass was varied from $25 \mathrm{t}$ to 50t. The low mass and high mass transition points described above were explicitly identified on the continuum of habitat masses. For this flight opportunity, a habitat mass of less than $30 \mathrm{t}$ will result in a reduction in the number of SLS launches while a habitat mass greater than $41.8 \mathrm{t}$ will result in an increase in the number of SLS launches. Due to variations in the trajectories and resulting $\Delta \mathrm{V}$ budgets for each flight opportunity, these transition points will vary between flight opportunities. It is also important to note that architecture approaches that universally reduce the $\Delta \mathrm{V}$ budget across all flight opportunities and higher-performing chemical propulsion stages that are less sensitive to payload mass will result in wider ranges of values for which no change in SLS launch requirements will result.

\section{SUMMARY AND CONCLUSIONS}

The SEP-Chem transportation architecture presented in this paper represents a viable transportation architecture for achieving the high-level goals of the Evolvable Mars Campaign study. The architecture takes advantage of the best aspects of both propulsion technologies to assemble a sustainable Mars exploration campaign leveraging the technology investments of today. High-efficiency SEP evolved from the investments made in the ARRM mission deliver cargo elements to Mars while high-thrust methane propulsion stages using engines common to those required for landing on Mars delivery the crew quickly and safely.

Several trades were performed to ensure that the right balance was struck between performance and programmatics. The SEP system's assumed power level delivers the most payload possible while remaining an evolved version of the ARRM spacecraft. Delivery is further maximized by using the SLS 2B launch vehicle. The globally common approach to the methane stage design supports reduced manufacturing costs while 
minimizing the performance impact. Unique aggregation orbits and trajectories ensure that energy requirements are minimal and enable a propulsive Earth return that supports the reuse of the transportation habitat and avoids costly upgrades to the Orion spacecraft. Overall, the SEP-Chem approach to transportation for the EMC provides a balanced approach that enables a steady programmatic progression that ultimately extends humanity's presence in the solar system and opens a new and exciting chapter in human exploration.

\section{REFERENCES}

[1] Smith, B., Nazario, M, and Manzella, D, "Advancement of a 30kW Solar Electric Propulsion System Capability for NASA Human and Robotic Exploration Missions," IAC-12-C4.4.2, 63rd International Astronautical Congress, Naples, Italy, October 2012.

[2] Brophy, John R., Muriel Noca, "Electric Propulsion for Solar System Exploration," JOURNAL OF PROPULSION AND POWER, Vol. 14, No. 5, September -October 1998.

[3] Welander, Benjamin, Jeff Monheiser, Nicole Meckel, Kristi de Grys and Peter Peterson, Vadim Khayms, "Demonstration of the XR-12 Hall Current Thruster," EPC-2013-451, Presented at the 33rd International Electric Propulsion Conference, The George Washington University, Washington, D.C., USA October 6-10, 2013.

[4] Drake, Bret, ed. "Human Exploration of Mars Design Reference Architecture 5.0”, NASA SP-2009-566, July, 2009.

\section{BIOGRAPHIES}

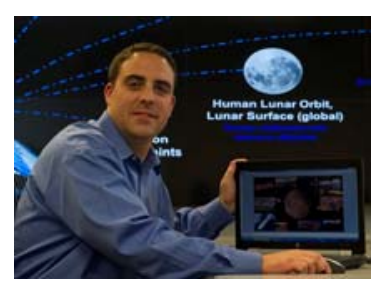

Tom Percy is an aerospace engineer in the Advanced Concepts Office at Marshall Space Flight Center. Mr. Percy has supported NASA's human and robotic space exploration programs for over 10 years in the areas of advanced propulsion and mission architecture analysis. Mr. Percy's focus areas include spacecraft and mission concept development, technology assessment, trajectory analysis and advanced propulsion systems. Mr. Percy holds a Bachelor of Science in Mechanical Engineering from Rochester Institute of Technology, a Master of Science in Aerospace Engineering from Georgia Institute of Technology, and is currently pursuing a PhD in Aerospace Systems Engineering at the University of Alabama in Huntsville.

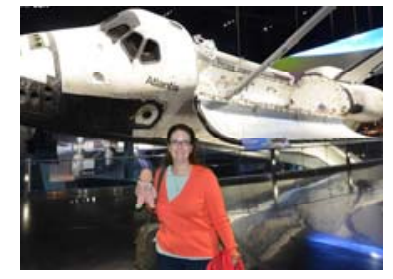

Melissa L. McGuire is currently serving as the deputy for mission design for the Asteroid Redirection Robotic Mission (ARRM) and has spent her over 20 year career at NASA Glenn Research Center as a trajectory mission analyst and a spacecraft system integration analyst for human and robotic future NASA exploration and science focused missions. In addition to mission design, Ms. McGuire is the deputy of and a founding member of the concurrent design team at NASA GRC known as COMPASS. Ms. McGuire holds a Master of Science in Aerospace Engineering from the University of Cincinnati and a Bachelor of Science in Engineering Physics from John Carroll University.

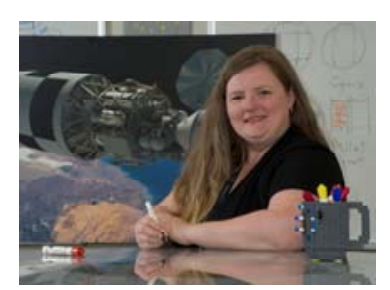

Tara Polsgrove is an aerospace engineer in the Flight Programs and Partnerships Office at NASA's Marshall Space Flight Center. She has been with NASA since 2000 and has worked on many conceptual designs of advanced spacecraft, including performance and vehicle integration for the Altair Lunar Lander. Her background is in interplanetary trajectory optimization and mission analysis. Recent work has focused on Mars transportation and lander designs supporting missions to send humans to Mars. Ms. Polsgrove has a Bachelor of Science in Aerospace Engineering from the Georgia Institute of Technology and a Master of Science in Engineering with a Systems Engineering focus from the University of Alabama in Huntsville. 